\title{
Three-dimensional dispersive metallic photonic crystals with a bandgap and a high cutoff frequency
}

\author{
Ma Luo and Qing Huo Liu* \\ Department of Electrical and Computer Engineering, Duke University, Durham, North Carolina 27708, USA \\ *Corresponding author: qhliu@duke.edu
}

Received April 7, 2010; revised June 8, 2010; accepted June 18, 2010;

posted June 30, 2010 (Doc. ID 126636); published July 30, 2010

\begin{abstract}
The goal of this work is to analyze three-dimensional dispersive metallic photonic crystals (PCs) and to find a structure that can provide a bandgap and a high cutoff frequency. The determination of the band structure of a PC with dispersive materials is an expensive nonlinear eigenvalue problem; in this work we propose a rational-polynomial method to convert such a nonlinear eigenvalue problem into a linear eigenvalue problem. The spectral element method is extended to rapidly calculate the band structure of three-dimensional PCs consisting of realistic dispersive materials modeled by Drude and Drude-Lorentz models. Exponential convergence is observed in the numerical experiments. Numerical results show that, at the low frequency limit, metallic materials are similar to a perfect electric conductor, where the simulation results tend to be the same as perfect electric conductor PCs. Band structures of the scaffold structure and semi-woodpile structure metallic PCs are investigated. It is found that band structures of semi-woodpile PCs have a very high cutoff frequency as well as a bandgap between the lowest two bands and the higher bands. (C) 2010 Optical Society of America

OCIS codes: $000.4430,160.5298$.
\end{abstract}

\section{INTRODUCTION}

Three-dimensional (3-D) photonic crystals (PCs) consisting of dispersive materials are a very interesting research topic [1-3]. Usually dispersive materials are metallic, so these PCs can be called metallic PCs. The band structures of 3-D metallic PCs have quite different properties from 3-D PCs consisting of dielectric materials. One of the most important differences is the existence of a cutoff frequency in some 3-D metallic PCs depending on the spatial structure of the material inside the unit cell of the PCs. For example, a 3-D wire mesh PC was designed to have a high cutoff frequency [2]. Another example of PCs that have a high cutoff frequency is the face-centered-cubic lattice of dielectric spheres embedded in a dispersive background material [4]. It seems that the condition for the existence of a cutoff frequency is the connectivity of the dispersive material. Many efforts focus on making use of 3-D metallic PCs to construct metamaterials [5,6], lefthanded materials [7], and waveguides [8].

The goal of this work is to analyze 3-D dispersive metallic PCs and to find a structure that can provide a bandgap and a high cutoff frequency. Previously, band structures of 3-D metallic PCs have been calculated by a timedomain simulation method in [9]. It is well known that time-domain simulation methods need to take a long simulation time to extract the band structures of PCs because of their strong resonance. Another group uses the Korringa-Kohn-Rostocker method [10,11] to calculate the band structures in the frequency domain. However, up until now this method is restricted to PCs consisting of non-overlapping spheres; either the sphere or the background can be a dispersive material in this work. Another method to calculate the band structure of a dispersive PC is to solve the nonlinear eigenvalue problem by an iterative method, by considering the frequency dependence of the permittivity of the metal [4]. The performance of this method strongly depends on the initial guess of the solution. In the research of another group, the auxiliary field is introduced in the frequency domain to model the dispersive materials so that the nonlinear eigenvalue problem is transformed into a linear eigenvalue problem $[12,13]$. In order to develop a general and efficient tool to calculate the band structures of 3-D metallic PCs, we propose a rational-polynomial method to convert the nonlinear eigenvalue problem into a linear one, and we extend the spectral element method (SEM) to model 3-D dielectric PCs with dispersive materials. The rationalpolynomial method is equivalent to the introduction of frequency-domain auxiliary fields in [12].

The SEM exhibits very high accuracy and efficiency in solving various fields [14-18]. This method solves the frequency-domain full vector wave equation of electromagnetic fields by discretizing the system into a matrix equation and solves the resultant matrix eigenvalue problem. We have shown that the SEM has exponential convergence for two-dimensional (2-D) and 3-D PCs consisting of dielectric materials $[19,20]$. In the calculation of band structures of PCs consisting of dispersive materials, the matrix equation becomes a nonlinear eigenvalue equation. Previously, the SEM for 2-D PCs has been extended to calculate the 2-D metallic PCs [21] by solving the nonlinear eigenvalue equation iteratively through the Brent method. This method can also be used for 3-D metallic PCs, but the computational time will be very long 
because the number of unknowns in the matrix equation of 3-D PCs is much larger than for 2-D PCs. Furthermore, as mentioned above, the iterative solution strongly depends on the initial guess. For 3-D metallic PCs, as the band structures are more complicated and contain more degeneracy, it is much more difficult to find a good initial guess. In this paper, we use another scheme to solve the nonlinear eigenvalue equation for 3-D metallic PCs by assuming that the dispersive material is described by the Drude or (and) Lorentz model [22,23]. Since the permittivity of the Drude or (and) Lorentz model is a rationalpolynomial function of frequency, the nonlinear eigenvalue problem can be transformed into a linear eigenvalue problem with a larger size. In this way, the band structures of 3-D metallic PCs with a complicated spatial structure can be calculated efficiently.

In this paper, several 3-D metallic PCs with complicated spatial structures inside the unit cell with a large cutoff frequency are designed and simulated, including the scaffold structure and semi-woodpile structure. It is found that the semi-woodpile 3-D metallic PCs have a large cutoff frequency as well as a bandgap between the lowest two bands and the higher bands. Since there is a cutoff frequency in a 3-D metallic PC, it is always possible to design a cavity or waveguide with the resonance frequency lower than the cutoff frequency so that the low frequency photon cannot propagate in the 3-D metallic PC. The bandgap above the cutoff frequency can also support another cavity or waveguide mode. Considering the gap between zero frequency and the cutoff frequency as one bandgap, it is thus possible to design a cavity of waveguide with two resonance frequencies to yield a double bandgap system. The double resonance frequencies are very useful for the investigation of many optical effects in confined optical systems, such as electromagnetically induced transparency and Raman scattering [24].

\section{DISPERSIVE MATERIALS AND THE SPECTRAL ELEMENT METHOD}

The objective of a simulation method for PCs with dispersive anisotropic materials is to solve for electromagnetic fields described by the full vector Helmholtz equations,

$$
\begin{aligned}
& \nabla \times\left[\mu_{r}^{-1}(\nabla \times \mathbf{E})\right]-k_{0}^{2} \varepsilon_{r} \mathbf{E}=0, \\
& \nabla \times\left[\varepsilon_{r}^{-1}(\nabla \times \mathbf{H})\right]-k_{0}^{2} \mu_{r} \mathbf{H}=0,
\end{aligned}
$$

where $k_{0}=\omega / c$ is the wave number in vacuum, and $\varepsilon_{r}$ and $\mu_{r}$ are the $3 \times 3$ relative permittivity and relative permeability tensors that can be frequency dependent. In a 3-D $\mathrm{PC}$, the Bloch periodic boundary condition is

$$
\begin{aligned}
& \mathbf{E}_{\|}(\mathbf{r}+\mathbf{R})=\mathbf{E}_{\|}(\mathbf{r}) e^{-j \mathbf{k} \cdot \mathbf{R}}, \\
& \mathbf{H}_{\|}(\mathbf{r}+\mathbf{R})=\mathbf{H}_{\|}(\mathbf{r}) e^{-j \mathbf{k} \cdot \mathbf{R}},
\end{aligned}
$$

where $\mathbf{E}_{\|}\left(\mathbf{H}_{\|}\right)$is the tangential component of electric (magnetic) field at the boundary of the unit cell, and $\mathbf{r}$ and $\mathbf{r}+\mathbf{R}$ are position vectors at the opposite sides of the boundary with $\mathbf{R}$ being written as

$$
\mathbf{R}=l \mathbf{a}_{1}+m \mathbf{a}_{2}+n \mathbf{a}_{3} .
$$

Here $\left(\mathbf{a}_{1}, \mathbf{a}_{2}, \mathbf{a}_{3}\right)$ are the three primitive lattice vectors of the unit cell, $(l, m, n)$ are three integers, and $\mathbf{k}$ is the Bloch wave vector. When the material in the unit cell of a $\mathrm{PC}$ is dispersive, the relative permittivity $\varepsilon_{r}$ depends on frequency (or equivalently depends on the wave number in vacuum). One kind of dispersive material is described by the Drude model, which gives the relation between the relative permittivity and frequency as

$$
\varepsilon_{r}\left(k_{0}\right)=1-\frac{k_{P}^{2}}{k_{0}^{2}-i \Gamma_{P} k_{0}},
$$

where the plasmon wave number $k_{P}=\omega_{P} / c$ is related to the plasmon frequency $\omega_{P}$, and $\Gamma_{P}$ is the corresponding damping frequency. For some realistic materials, the frequency dependence of permittivity is more complicated. For example, we consider metallic materials such as gold, whose permittivity is modeled by the Drude-Lorentz model given by $[22,23]$. The relative permittivity is written as

$$
\begin{aligned}
\varepsilon_{r}\left(k_{0}\right)= & \varepsilon_{\mathrm{inf}}-\frac{k_{P}^{2}}{k_{0}^{2}-i \Gamma_{P} k_{0}}+\sum_{j=1,2} \frac{A_{j}}{\sqrt{2}}\left[\frac{1-i}{k_{j}-k_{0}-\Gamma_{j} i}\right. \\
& \left.+\frac{1+i}{k_{j}+k_{0}+\Gamma_{j} i}\right],
\end{aligned}
$$

where $\varepsilon_{\text {inf }}=1.54$ is the relative permittivity at infinitely large frequency for the Drude model; $k_{P}=(2 \pi / 143) \mathrm{nm}^{-1}$ and $\Gamma_{P}=(2 \pi / 14,500) \mathrm{nm}^{-1}$ are the Drude model parameters; and $A_{1}=1.27, \quad k_{1}=(2 \pi / 470) \mathrm{nm}^{-1}, \quad \Gamma_{1}$ $=(2 \pi / 1900) \mathrm{nm}^{-1}, A_{2}=1.1, k_{2}=(2 \pi / 325) \mathrm{nm}^{-1}$, and $\Gamma_{2}$ $=(2 \pi / 1060) \mathrm{nm}^{-1}$ are the parameters for the two pairs of poles in the Lorentz model. Other metals may have more pairs of poles in the Lorentz model, but the same principle proposed here applies.

The SEM solves either Eq. (1) or Eq. (2) by meshing the unit cell with non-overlapping curvilinear hexahedral elements and by expanding the field in terms of interpolation basis functions. In the reference domain, the basis functions $\hat{\boldsymbol{\Phi}}_{j}$ are constructed by Gauss-Lobatto-Legendre polynomials, which give a high accuracy interpolation. And then the basis functions are mapped to the real space of each element by a covariant mapping $\boldsymbol{\Phi}_{j}=\mathbf{J}^{-1} \hat{\boldsymbol{\Phi}}_{j}(\mathbf{J}$ is the Jacobian matrix), where $\boldsymbol{\Phi}_{j}$ is the $j$ th vector basis function. The covariant mapping keeps the tangential components of the basis functions continuous across the interface between adjacent elements. Because the tangential components of electric field are continuous, the electric field can be expanded by these basis functions as

$$
\mathbf{E}=\sum_{j=1}^{N} E_{j} \boldsymbol{\Phi}_{j},
$$

where $E_{j}$ is the expansion coefficient. After the process of the regular finite element method discretization, the vector Helmholtz equation is converted into the matrix equation 


$$
\mathbf{S E}=k_{0}^{2} \mathbf{M E},
$$

where $\mathbf{E}=\left[E_{1}, E_{2}, \ldots, E_{N}\right]^{T}$ is the column vector of the expansion coefficients, and $\mathbf{S}$ and $\mathbf{M}$ are the stiffness and mass matrices given by

$$
\mathbf{S}=\sum_{e=1}^{N_{e}} \mathbf{S}^{(e)}, \quad \mathbf{M}=\sum_{e=1}^{N_{e}} \mathbf{M}^{(e)}
$$

with elemental stiffness and mass matrices

$$
\begin{gathered}
S_{j, k}^{(e)}=\int_{\Omega_{e}} d \mathbf{r}\left(\nabla \times \boldsymbol{\Phi}_{j}\right) \cdot \mu_{r}^{-1} \cdot\left(\nabla \times \boldsymbol{\Phi}_{k}\right), \\
M_{j, k}^{(e)}=\int_{\Omega_{e}} d \mathbf{r} \boldsymbol{\Phi}_{j} \cdot \varepsilon_{r} \cdot \boldsymbol{\Phi}_{k},
\end{gathered}
$$

where $\Omega_{e}$ is the region of the eth element and $N_{e}$ is the total number of elements in the unit cell. Note that for a dispersive PC, Eq. (9) is a nonlinear eigenvalue problem because $\mathbf{M}$ or $\mathbf{S}$ depends on the frequency. To avoid solving this nonlinear eigenvalue problem by an expensive iterative method, we will transform this nonlinear problem into a linear problem.

To convert this nonlinear eigenvalue into a linear one, we assume that the medium inside the unit cell is piecewise homogeneous so that each element will have one homogeneous (but possibly dispersive) material. Since some elements are filled with homogeneous non-dispersive materials while others are filled with a homogeneous dispersive material, we need to separate elemental mass matrices of these different types of elements to allow subsequent transformation from a nonlinear eigenvalue problem into a linear one. To this end, the mass matrix is split into two matrices

$$
\mathbf{M}=\mathbf{M}_{n}+\varepsilon_{r}^{d}\left(k_{0}\right) \mathbf{M}_{d},
$$

where the subscript $n(d)$ denotes that for non-dispersive (dispersive) materials,

$$
\mathbf{M}_{n}=\sum_{e_{n}=1}^{N_{e}^{n}} \mathbf{M}^{\left(e_{n}\right)}, \quad \mathbf{M}_{d}=\sum_{e_{d}=1}^{N_{e}^{d}} \mathbf{M}^{\left(e_{d}\right)} / \varepsilon_{r}^{d}\left(k_{0}\right),
$$

and $N_{e}^{n}\left(N_{e}^{d}\right)$ is the number of non-dispersive (dispersive) elements. Then, Eq. (9) becomes

$$
\mathbf{S E}=k_{0}^{2} \mathbf{M}_{\mathbf{n}} \mathbf{E}+k_{0}^{2} \varepsilon_{r}^{d}\left(k_{0}\right) \mathbf{M}_{\mathbf{d}} \mathbf{E} .
$$

Here it is assumed that there is only one kind of isotropic dispersive material in the unit cell. When there is more than one kind of dispersive material or the dispersive material is anisotropic, the mass matrix is further split. Since the relative permittivity depends on frequency, which is the eigenvalue in Eq. (15), the equation becomes a nonlinear eigenvalue equation. If the permittivity of the dispersive material is a rational-polynomial function of frequency, Eq. (15) can be transformed into a linear eigenvalue equation with a larger size. We discuss four situations where the dispersive material is (a) a lossless Drude model, (b) a lossy Drude model, (c) a PEC, and (d) a realistic metal such as gold.

\section{A. Lossless Drude Model}

When the dispersive material is a lossless Drude model material, the relative permittivity is

$$
\varepsilon_{r}\left(k_{0}\right)=1-\frac{k_{P}^{2}}{k_{0}^{2}} .
$$

Inserting Eq. (16) into Eq. (15) and multiplying the equation with $k_{0}^{2}$, we have

$$
\left(\mathbf{S}+k_{P}^{2} \mathbf{M}_{\mathbf{d}}\right) \mathbf{E}=k_{0}^{2}\left(\mathbf{M}_{\mathbf{n}}+\mathbf{M}_{\mathbf{d}}\right) \mathbf{E} .
$$

This becomes a linear eigenvalue problem with the same size as Eq. (15), and thus can be solved with the same cost as the problem with non-dispersive materials.

\section{B. Lossy Drude Model}

If the dispersive material is lossy and is described by Eq. (6), we multiply Eq. (15) with $k_{0}^{2}-i \Gamma_{P} k_{0}$ and collect the matrices with the same power of coefficient $k_{0}$ to arrive at a third-order nonlinear eigenvalue problem with $k_{0}$ being the eigenvalue instead of $k_{0}^{2}$,

$$
\begin{aligned}
& {\left[i \Gamma_{P} \mathbf{S}-k_{0}\left(k_{P}^{2} \mathbf{M}_{\mathbf{d}}+\mathbf{S}\right)-i \Gamma_{P} k_{0}^{2}\left(\mathbf{M}_{\mathbf{n}}+\mathbf{M}_{\mathbf{d}}\right)+k_{0}^{3}\left(\mathbf{M}_{\mathbf{n}}+\mathbf{M}_{\mathbf{d}}\right)\right] \mathbf{E}} \\
& \quad=0 .
\end{aligned}
$$

Defining $\mathbf{F}=k_{0} \mathbf{E}$ and $\mathbf{G}=k_{0} \mathbf{F}$, and inserting them into Eq. (18), together with the definition, we have a linear eigenvalue problem of triple size,

$$
\begin{gathered}
{\left[\begin{array}{ccc}
0 & \mathbf{I} & 0 \\
0 & 0 & \mathbf{I} \\
i \Gamma_{P} \mathbf{S} & -\left(k_{P}^{2} \mathbf{M}_{\mathbf{d}}+\mathbf{S}\right) & -i \Gamma_{P}\left(\mathbf{M}_{\mathbf{n}}+\mathbf{M}_{\mathbf{d}}\right)
\end{array}\right]\left[\begin{array}{l}
\mathbf{E} \\
\mathbf{F} \\
\mathbf{G}
\end{array}\right]} \\
\quad=k_{0}\left[\begin{array}{ccc}
\mathbf{I} & 0 & 0 \\
0 & \mathbf{I} & 0 \\
0 & 0 & \mathbf{M}_{\mathbf{n}}+\mathbf{M}_{\mathbf{d}}
\end{array}\right]\left[\begin{array}{l}
\mathbf{E} \\
\mathbf{F} \\
\mathbf{G}
\end{array}\right],
\end{gathered}
$$

where $\mathbf{I}$ is an identity matrix.

\section{Perfect Electric Conductor (PEC) Model}

When the frequency is much smaller than the plasma frequency, the relative permittivity is negative with a very large amplitude. The skin depth becomes so small that the electromagnetic wave hardly penetrates into the dispersive material. In this limit, the dispersive material can be modeled as a PEC. In order to calculate the band structure of a 3-D PC consisting of a PEC, we only need to set the unknown electric field at the nodal points located inside and on the surface of the PEC with tangential component to be zero. This is equivalent to reducing the stiffness and mass matrices from Eq. (9) to smaller matrices by removing the corresponding rows and columns. The resultant problem is thus a linear eigenvalue problem with fewer unknowns than for a non-dispersive dielectric PC.

\section{Realistic Materials Such as Gold}

If the dispersive material is a realistic metal such as gold with permittivity given by Eq. (7) in the optical frequency regime, the same process of multiplying Eq. (15) by all of the denominator terms is applied. The result is a seventhorder nonlinear eigenvalue problem, which is trans- 
formed into a linear eigenvalue problem with a size seven times as large. The final eigenvalue problem is given by

$$
\begin{gathered}
{\left[\begin{array}{ccccccc}
0 & \mathbf{I} & 0 & 0 & 0 & 0 & 0 \\
0 & 0 & \mathbf{I} & 0 & 0 & 0 & 0 \\
0 & 0 & 0 & \mathbf{I} & 0 & 0 & 0 \\
0 & 0 & 0 & 0 & \mathbf{I} & 0 & 0 \\
0 & 0 & 0 & 0 & 0 & \mathbf{I} & 0 \\
0 & 0 & 0 & 0 & 0 & 0 & \mathbf{I} \\
i \mathbf{T} & \mathbf{U} & i \mathbf{V} & \mathbf{W} & i \mathbf{X} & \mathbf{Y} & i \mathbf{Z}
\end{array}\right]\left[\begin{array}{c}
\mathbf{E} \\
\mathbf{F} \\
\mathbf{G} \\
\mathbf{H} \\
\mathbf{J} \\
\mathbf{K} \\
\mathbf{L}
\end{array}\right]} \\
= \\
{\left[\begin{array}{ccccccc}
\mathbf{I} & 0 & 0 & 0 & 0 & 0 & 0 \\
0 & \mathbf{I} & 0 & 0 & 0 & 0 & 0 \\
0 & 0 & \mathbf{I} & 0 & 0 & 0 & 0 \\
0 & 0 & 0 & \mathbf{I} & 0 & 0 & 0 \\
0 & 0 & 0 & 0 & \mathbf{I} & 0 & 0 \\
0 & 0 & 0 & 0 & 0 & \mathbf{I} & 0 \\
0 & 0 & 0 & 0 & 0 & 0 & \mathbf{M}_{\mathbf{n}}{ }^{\prime}
\end{array}\right]\left[\begin{array}{c}
\mathbf{E} \\
\mathbf{F} \\
\mathbf{G} \\
\mathbf{H} \\
\mathbf{J} \\
\mathbf{K} \\
\mathbf{L}
\end{array}\right],}
\end{gathered}
$$

where $\mathbf{M}_{\mathbf{n}}{ }^{\prime}=\mathbf{M}_{\mathbf{n}}+\varepsilon_{\text {inf }} \mathbf{M}_{\mathbf{d}}$, vectors $\mathbf{F}$ to $\mathbf{L}$ are defined at the first six rows of the equation, and matrices $\mathbf{T}$ to $\mathbf{Z}$ are defined as follows:

$$
\begin{gathered}
\mathbf{T}=\Gamma_{P} \chi_{1} \chi_{2} \mathbf{S} \\
\mathbf{U}=\left(\chi_{1} \chi_{2}+2 \Gamma_{P} \psi\right) \mathbf{S}+\chi_{1} \chi_{2} k_{P}^{2} \mathbf{M}_{\mathbf{d}}, \\
\mathbf{V}=-\left(\Gamma_{P} \kappa+2 \psi\right) \mathbf{S}-\Gamma_{P} \chi_{1} \chi_{2} \mathbf{M}_{\mathbf{n}}{ }^{\prime}-2\left(A_{2} \Gamma_{P} \chi_{1} \varphi_{2}+A_{1} \Gamma_{P} \chi_{2} \varphi_{1}\right. \\
\left.+\psi k_{P}^{2}\right) \mathbf{M}_{\mathbf{d}}, \\
\mathbf{W}=-\left(2 \Gamma_{P} \Gamma_{L}+\kappa\right) \mathbf{S}-\left(\chi_{1} \chi_{2}+2 \Gamma_{P} \psi\right) \mathbf{M}_{\mathbf{n}}{ }^{\prime}-\left[2 A _ { 1 } \left(\chi_{2} \varphi_{1}\right.\right. \\
\left.\left.+\Gamma_{P} \vartheta_{2}\right)+2 A_{2}\left(\chi_{1} \varphi_{2}+\Gamma_{P} \vartheta_{1}\right)+\kappa k_{P}^{2}\right] \mathbf{M}_{\mathbf{d}}, \\
\mathbf{X}=\left(\Gamma_{P}+2 \Gamma_{L}\right) \mathbf{S}+\left(\Gamma_{P} \kappa+2 \psi\right) \mathbf{M}_{\mathbf{n}}{ }^{\prime}+2\left\{A_{1}\left[\vartheta_{2}+\Gamma_{P}\left(\varphi_{1}+2 \Gamma_{2}\right)\right]\right. \\
\left.+A_{2}\left[\vartheta_{1}+\Gamma_{P}\left(\varphi_{2}+2 \Gamma_{1}\right)\right]+\Gamma_{L} k_{P}^{2}\right\} \mathbf{M}_{\mathbf{d}}, \\
\mathbf{Y}=\mathbf{S}+\left(\kappa+2 \Gamma_{P} \Gamma_{L}\right) \mathbf{M}_{\mathbf{n}}{ }^{\prime}+\left[2 A_{1}\left(\Gamma_{P}+2 \Gamma_{2}+\varphi_{1}\right)+2 A_{2}\left(\Gamma_{P}\right.\right. \\
\left.\left.+2 \Gamma_{1}+\varphi_{2}\right)+k_{P}^{2}\right] \mathbf{M}_{\mathbf{d}}, \\
\quad \mathbf{Z}=-\left(\Gamma_{P}+2 \Gamma_{L}\right) \mathbf{M}_{\mathbf{n}}{ }^{\prime}-2\left(A_{1}+A_{2}\right) \mathbf{M}_{\mathbf{d}} .
\end{gathered}
$$

In the above, $\Gamma_{L}=\Gamma_{1}+\Gamma_{2}, \varphi_{1,2}=\Gamma_{1,2}+k_{1,2}, \chi_{1,2}=\Gamma_{1,2}^{2}+k_{1,2}^{2}$, $\psi=\Gamma_{1}^{2} \Gamma_{2}+\Gamma_{1} \Gamma_{2}^{2}+\Gamma_{1} k_{2}^{2}+\Gamma_{2} k_{1}^{2}, \quad \kappa=\Gamma_{1}^{2}+4 \Gamma_{1} \Gamma_{2}+\Gamma_{2}^{2}+k_{1}^{2}+k_{2}^{2}, \quad \vartheta_{1}$ $=2 \Gamma_{1} \Gamma_{2}+\Gamma_{1}^{2}+k_{1}^{2}+2 \Gamma_{1} k_{2}$, and $\vartheta_{2}=2 \Gamma_{1} \Gamma_{2}+\Gamma_{2}^{2}+k_{2}^{2}+2 \Gamma_{2} k_{1}$.

The above transformation converts the original nonlinear eigenvalue problem into a linear one. This approach is applicable to all those common metals reported in $[22,23,25]$.

\section{NUMERICAL RESULTS}

Two kinds of 3-D PC structures with a cubic lattice are investigated. The distributions of dispersive material blocks of $2 \times 2 \times 2$ unit cells are shown in Fig. 1(a) for the scaffold structure and in Fig. 1(b) for the semi-woodpile structure. It is found that both these structures have a high cutoff (a)

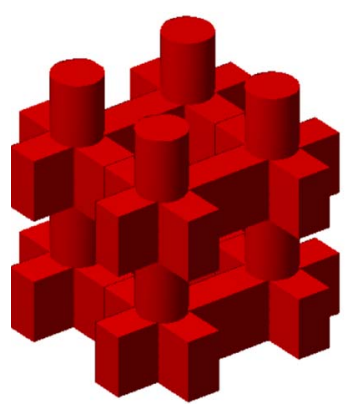

(b)

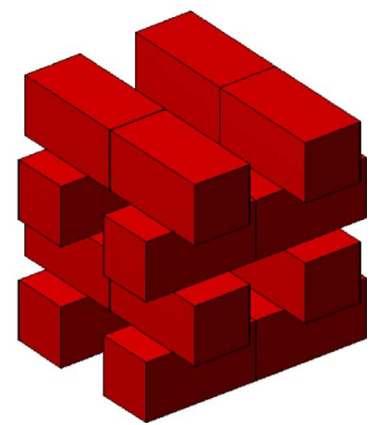

Fig. 1. (Color online) The PC structure of $2 \times 2 \times 2$ unit cells under investigation. (a) Scaffold structure with a circular cylinder in the middle. (b) Semi-woodpile structure.

frequency. Since the losses of most dispersive metallic materials are very small, the lossy Drude model will give a very similar band structure as the lossless Drude model. Hence we do not show the numerical results of the lossy Drude model here. Only the numerical results of the lossless Drude model, PEC model, and gold are shown and compared below. For each kind of 3-D PCs, the investigation starts with the band structure of a Drude model PC, because this model is a good approximation for most realistic metallic materials. The band structures are determined by both plasmon wave number and size of the unit cell, but the normalized band structures only depend on $k_{P} a / 2 \pi$ so that the normalized band structures of various values of $k_{P} a / 2 \pi$ are investigated. Specifically, a PEC has $k_{P} a / 2 \pi \rightarrow \infty$, which can be recognized as the size of the unit cell being much larger than the plasmon wavelength $\lambda_{P}=2 \pi / k_{P}$. Most of the characteristics of the normalized band structures are preserved in this extreme situation. In addition, the band structures of the PC consisting of gold with various sizes of a unit cell are shown. Most realistic materials, such as gold, are different from the Drude model only by a few poles of Lorentz models with small amplitudes. This small modification has small effects on the normalized band structures.

\section{A. Scaffold Structure PC}

The scaffold PC shown in Fig. 1(a) has a unit cell of $a$ $\times a \times a$. The dimensions inside one unit cell are the height of the circular cylinder and the two square blocks are $0.5 a$, the radius of the circular cylinder is $0.2 a$, and the width of the two square blocks is $0.2 \sqrt{2} a$. The material in the background (not shown) is air. The band structures of this scaffold structure PC consisting of a Drude model dispersive material with (a) $k_{P} a / 2 \pi=1$, (b) $k_{P} a / 2 \pi=2$, (c) $k_{P} a / 2 \pi=3$, and (d) PEC are plotted in Figs. 2(a)-2(d), respectively. All four band structures have a cutoff frequency. Around the surface plasmon frequency $k_{P} / \sqrt{2}$, the band structures of the Drude model PCs become very dense because of the existence of surface plasmon modes. When the plasmon frequency becomes higher, as indicated in Figs. 2(a)-2(c), the cutoff frequency becomes higher and gets closer to the cutoff frequency of the PEC PC. This observation is consistent with the fact that a material with an infinitely large plasmon frequency approaches a PEC. The similarity of the shape of the lowest 
(a)

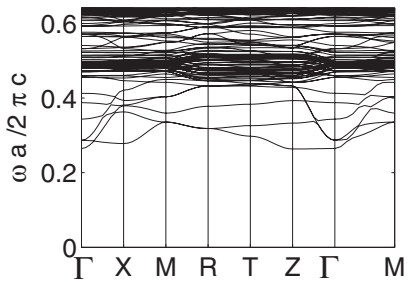

(c)

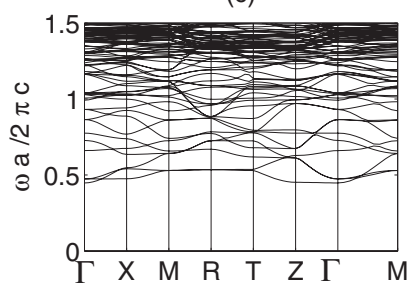

(b)

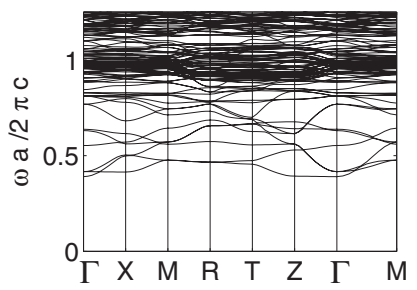

(d)

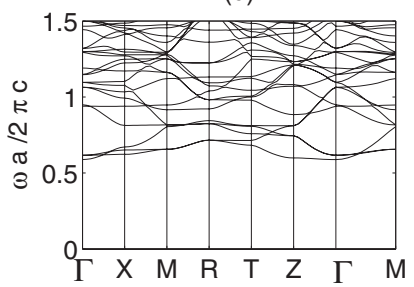

Fig. 2. Band structure of the scaffold structure PC in Fig. 1(a) with the Drude model dispersive materials with (a) $k_{P} a / 2 \pi=1$, (b) $k_{P} a / 2 \pi=2$, (c) $k_{P} a / 2 \pi=3$, and (d) PEC, where $a$ is the length of a unit cell size. The $x$-axis is the Bloch wave vector that scales through the high symmetry points in the first Brillouin zone denoted by $\Gamma, \mathrm{X}, \mathrm{M}, \mathrm{R}, \mathrm{T}$, and $\mathrm{Z}$, where the normalized wave number vectors are given by $\mathbf{k}=(0,0,0),(1 / 2,0,0),(1 / 2,1 / 2,0),(1 / 2,1 / 2,1 / 2)$, $(0,1 / 2,1 / 2)$, and $(0,0,1 / 2)$, respectively.

few bands between Drude model PCs and a PEC PC also increases as the plasmon frequency becomes higher. The high cutoff frequency is expected because of the connectivity of the spatial structure of the dispersive material.

The real part of the band structure of the 3-D scaffold structure PC consisting of gold is shown in Fig. 3, with the lattice sizes $a=400 \mathrm{~nm}$ [Fig. 3(a)] and $a=800 \mathrm{~nm}$ [Fig. 3(b)]. In order to make it easier to compare to experiments, the $Y$-axis is scaled by $\omega / 2 \pi c$, or one over wavelength, and the cutoff frequency is transformed into the cutoff wavelength. The figures show that the cutoff wavelengths are smaller than $1100 \mathrm{~nm}$ for the lattice size $a$ $=400 \mathrm{~nm}$ and are smaller than $1700 \mathrm{~nm}$ for the lattice size $a=800 \mathrm{~nm}$. Since gold is lossy, there are no very dense surface plasmon modes as shown in the lossless Drude model PCs. The imaginary part of the band structure is smaller than $0.009 \omega a / 2 \pi c$ throughout the whole band structure. (a)

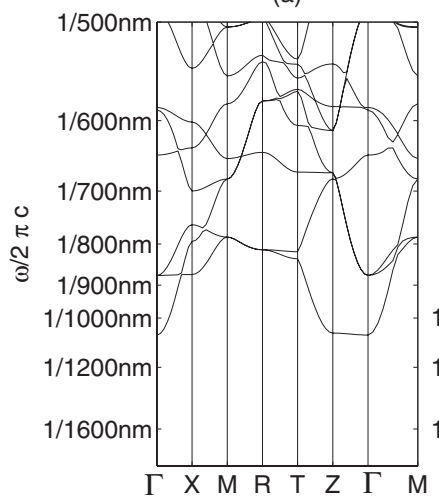

(b)

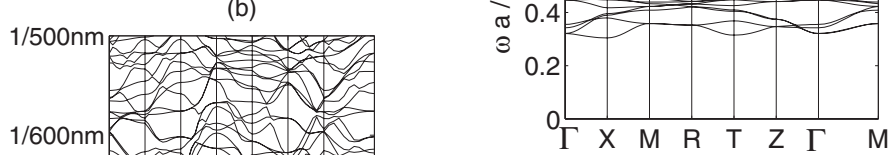

(c)

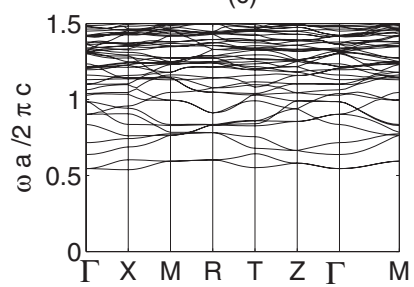

(a)

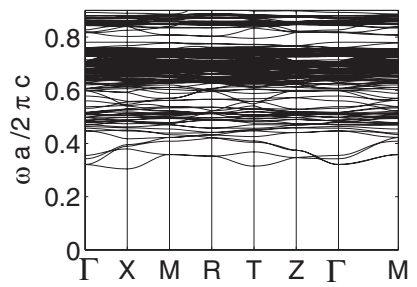

$1 / 700 \mathrm{~nm} \rightleftharpoons$

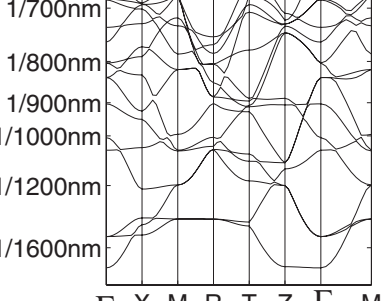

B. Semi-Woodpile Structure PC

The semi-woodpile structure PC in Fig. 1(b) is also a cubic lattice with lattice size $a \times a \times a$. The width and height of each block are both $0.5 a$. Figure 4 shows the band structure of this PC structure consisting of a Drude model material with $k_{P} a / 2 \pi=1$ [Fig. 4(a)], $k_{P} a / 2 \pi=2$ [Fig. 4(b)], $k_{P} a / 2 \pi=3$ [Fig. 4(c)], and PEC [Fig. 4(d)]. A higher cutoff frequency than the scaffold structure PC is shown. The lowest several bands of the Drude model PC with a large plasmon frequency, shown in Fig. 4(c), are similar to the lowest several bands of a PEC PC in Fig. 4(d). From Fig. 4(d), the band structure of the PEC PC has a bandgap above the lowest two bands besides the cutoff frequency. The real part of the band structure of the PC consisting of gold is plotted in Fig. 5, with the lattice sizes being 400 $\mathrm{nm}$ [Fig. 5(a)] and $800 \mathrm{~nm}$ [Fig. 5(b)]. The cutoff wavelength is less than 800 and $1300 \mathrm{~nm}$, respectively. When the lattice size is $a=800 \mathrm{~nm}$, a bandgap is opened up between the lowest two bands and the higher bands. The decay times of the lowest two bands in the band structures (a) and (b), which are defined as $2 \pi / \operatorname{Im}(\omega)$, are plotted in Figs. 5(c) and 5(d), respectively. The decay time of the PC with a lattice size $a=800 \mathrm{~nm}$ is larger than $0.5 \mathrm{ps}$, and the center of the bandgap above the first two bands is $0.7435 \times 2 \pi c / a$ which has a period of $0.0036 \mathrm{ps}$; thus, this decay time is more than 139 times larger than the period of the photonic state in the bandgap. Therefore, this PC is equivalent to a homogeneous dielectric material with a loss tangent smaller than 0.014 at the optical frequency.

Because the band structures in Figs. 4(d) and 5(b) have a small bandgap above the lowest two bands, we investigate the relative numerical error of these two band structures so that the existence of this bandgap could be confirmed within the accuracy of the numerical data. The relative error of the first and third bands with the normalized wave vector being at the $R$ point $\mathbf{k}$ $=(1 / 2,1 / 2,1 / 2)$ of the first Brillouin zone versus the order of the SEM is plotted in Fig. 6. The relative error at other wave vector positions in the first Brillouin zone is at a similar level. Figure 6(a) is the relative error of the PEC

Fig. 3. Band structure of the scaffold structure PC in Fig. 1(a) with gold as the material. The sizes of the unit cells are (a) $a$ $=400 \mathrm{~nm}$ and (b) $a=800 \mathrm{~nm}$.
Fig. 4. Band structure of the semi-woodpile structure $\mathrm{PC}$ in Fig. 1(b) with the dispersive materials being Drude model material with (a) $k_{P} a / 2 \pi=1$, (b) $k_{P} a / 2 \pi=2$, (c) $k_{P} a / 2 \pi=3$, and (d) PEC, where $a$ is the length of a unit cell size. 
(a)

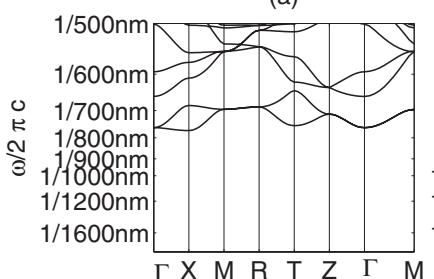

(c)

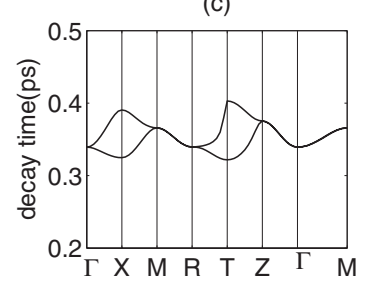

(b)

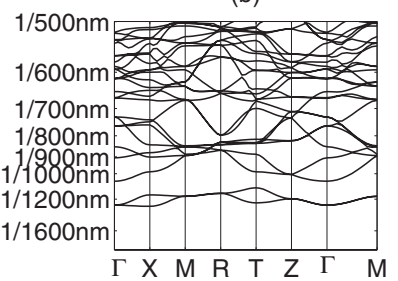

(d)

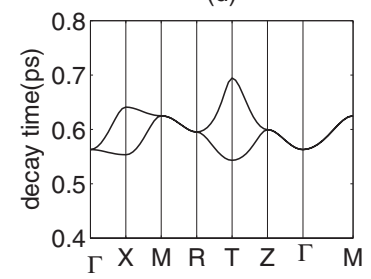

Fig. 5. Band structure of the semi-woodpile structure $\mathrm{PC}$ in Fig. 1(b) with gold as the material. The sizes of the unit cells are (a) $a=400 \mathrm{~nm}$ and (b) $a=800 \mathrm{~nm}$.

semi-woodpile PC, which shows an exponential convergence behavior. Since the PEC PC system yields a matrix equation with a fewer unknowns, we have results up to the eighth-order SEM. The relative error of the $i$ th-order SEM is obtained by comparing it with the result of the eighth-order SEM. From Fig. 6(a), the fourth-order SEM has a relative error smaller than $0.5 \times 10^{-2}$. The center of the bandgap is $0.8789 \times 2 \pi c / a$, then the absolute error around the bandgap is smaller than $0.0088 \times 2 \pi c / a$, which is much smaller than the width of the bandgap, $0.0891 \times 2 \pi c / a$. Therefore, the existence of the bandgap in Fig. 4(d) is confirmed.

For the gold semi-woodpile PC with a lattice size $a$ $=800 \mathrm{~nm}$, the relative error is plotted in Fig. 6(b). The matrix equation for this system has much more unknowns; thus we only have results up to the fifth-order SEM. The frequency dependence of the relative permittivity of gold is complicated; thus solving the nonlinear eigenvalue equation is more challenging and the relative

(a)

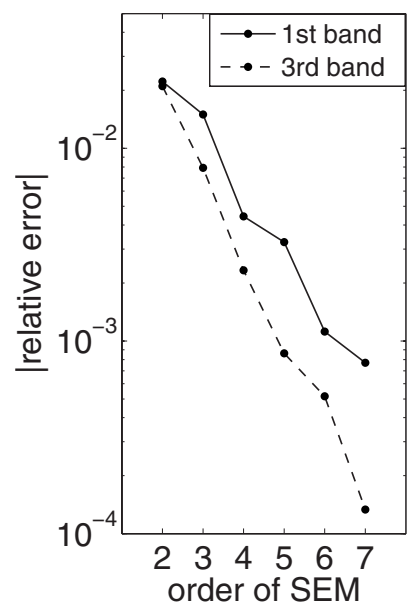

(b)

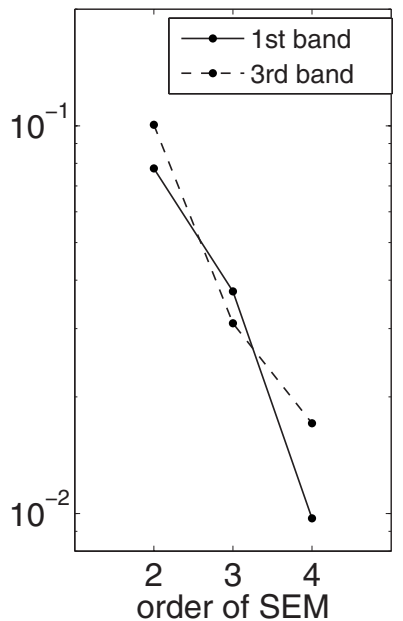

Fig. 6. Relative error of the SEM result at the $R$ point of the band structure of (a) PEC semi-woodpile PC in Fig. 4(d) and (b) gold semi-woodpile PC with lattice size $a=800 \mathrm{~nm}$ in Fig. 5(b), versus the order of the SEM.

error becomes larger. Nevertheless, the convergent behavior of the numerical result is still exponential, although with a slower speed of convergence. The band structure in Fig. 5(b) is given by the fourth-order SEM, whose relative error is smaller than $2 \times 10^{-2}$ from Fig. $6(\mathrm{~b})$. The center of the bandgap is $0.7435 \times 2 \pi c / a$; thus the absolute error around the bandgap is smaller than $0.0297 \times 2 \pi c / a$, which is smaller than the width of the bandgap 0.0346 $\times 2 \pi c / a$. As a result, the existence of the bandgap in Fig. $5(b)$ is also confirmed.

\section{DISCUSSION AND CONCLUSION}

Woodpile PCs consisting of dielectric materials are one of the most widely investigated PCs because they have a large bandgap [26-28]. We have calculated band structures of woodpile PCs consisting of various kinds of dispersive materials (although due to space limit not all have been shown here). It is found that the cutoff frequency of woodpile metallic PCs is smaller than that of the semi-woodpile metallic PCs, and woodpile metallic PCs do not have a second bandgap.

Since the semi-woodpile structure is simpler than the woodpile structure, we focus our investigation on semiwoodpile metallic PCs. As we can observe from Figs. 4(d) and 5(b), there is a bandgap as well as a high cutoff frequency. If the cutoff frequency is also counted as a bandgap between the lowest band and the zero frequency, this system has double bandgaps. At microwave frequencies, metallic materials usually behave as PECs, so we discuss the result for a PEC in Fig. 4(d) first. The bandgap under the cutoff frequency is very large. The cutoff frequency can be as high as $0.7 \omega a / 2 \pi c$. A high- $Q$ cavity mode with a frequency below the cutoff frequency could be easily constructed because of the large stop band. Since there is a second bandgap opened up at the frequency around $0.8 \omega a / 2 \pi c$, a second high- $Q$ cavity mode can exist through a proper design. As a result, a double cavitymode resonance system can be designed based on this PC.

At optical frequencies, metallic materials have more complicated behaviors because of their frequency dependent permittivity. However, realistic metallic materials, such as gold, can be approximated as a Drude model. When the lattice size $a$ is larger, the plasmon frequency becomes larger compared to the normalized frequency $2 \pi c / a$, so the materials become more similar to a PEC. From Fig. 5(a), when the lattice size is $400 \mathrm{~nm}$, the cutoff frequency is high, but there is no second bandgap. From Fig. 5(b), when the lattice size is $800 \mathrm{~nm}$, the cutoff frequency is still high, and the second bandgap is opened up. But the bandwidth of the second bandgap is smaller than that of a PEC PC. Therefore, it is more difficult to design the double cavity-mode resonance systems at optical frequencies. On the other hand, the PC with a lattice size of $800 \mathrm{~nm}$ could be useful as a propagation substrate for optical devices or optical filters because of the low loss tangent at optical frequencies.

In conclusion, we have extended the SEM method to calculate the band structures of 3-D dispersive metallic PCs. The metallic materials are modeled by the Drude model, PEC, and Drude-Lorentz model. The original nonlinear eigenvalue problem for such dispersive media has 
been converted into a linear eigenvalue problem through rational polynomials. Two configurations of $3-\mathrm{D} \mathrm{PCs}$, namely, the scaffold and semi-woodpile structures, consisting of all three kinds of dispersive materials, have been calculated. It is found that there is a cutoff frequency for both 3-D PC configurations. The semi-woodpile 3-D PCs open up the second bandgap when the dispersive material is a PEC or a Drude model material with a large plasmon frequency. This second bandgap provides a possibility to design a double cavity-mode resonance system, which is very useful for optical and microwave device applications [24].

\section{REFERENCES}

1. J. G. Fleming, S. Y. Lin, I. El-Kady, R. Biswas, and K. M. Ho, "All-metallic three-dimensional photonic crystals with a large infrared bandgap," Nature 417, 52-55 (2002).

2. D. F. Sievenpiper, M. E. Sickmiller, and E. Yablonovitch, "3D wire mesh photonic crystals," Phys. Rev. Lett. 76, 2480-2483 (1996).

3. I. El-Kady, M. M. Sigalas, R. Biswas, K. M. Ho, and C. M. Soukoulis, "Metallic photonic crystals at optical wavelengths," Phys. Rev. B 62, 15299-15302 (2000).

4. A. L. Pokrovsky, V. Kamaev, C. Y. Li, Z. V. Vardeny, A. L. Efros, D. A. Kurdyukov, and V. G. Golubev, "Theoretical and experimental studies of metal-infiltrated opals," Phys. Rev. B 71, 165114 (2005).

5. D. R. Smith, J. B. Pendry, and M. C. K. Wiltshire, "Metamaterials and negative refractive index," Science 305, 788-792 (2004).

6. S. Enoch, G. Tayeb, P. Sabouroux, N. Guérin, and P. Vincent, "A metamaterial for directive emission," Phys. Rev. Lett. 89, 213902 (2002).

7. P. V. Parimi, W. T. Lu, P. Vodo, J. Sokoloff, J. S. Derov, and S. Sridhar,"Negative refraction and left-handed electromagnetism in microwave photonic crystals," Phys. Rev. Lett. 92, 127401 (2004)

8. M. M. Sigalas, R. Biswas, K. M. Ho, C. M. Soukoulis, and D. D. Crouch, "Waveguides in three-dimensional metallic photonic band-gap materials," Phys. Rev. B 60, 4426-4429 (1999).

9. S. Fan, P. R. Villeneuve, and J. D. Joannopoulos, "Large omnidirectional band gaps in metallodielectric photonic crystals," Phys. Rev. B 54, 11245-11251 (1996).

10. A. Moroz, "Three-dimensional complete photonic-band-gap structures in the visible," Phys. Rev. Lett. 83, 5274-5277 (1999).

11. H. van der Lem, A. Tip, and A. Moroz, "Band structure of absorptive two-dimensional photonic crystals," J. Opt. Soc. Am. B 20, 1334-1341 (2003).

12. A. Tip, "Some mathematical properties of Maxwell's equations for macroscopic dielectrics," J. Math. Phys. 47, 012902 (2006)
13. J.-M. Combes, B. Gralak, and A. Tip, "Spectral properties of absorptive photonic crystals," in Waves in Periodic and Random Media, Vol. 339 of Contemporary Mathematics, P. Kuchment, ed. (American Mathematical Society, 2003), pp. $1-13$.

14. G. C. Cohen, Higher-order Numerical Methods for Transient Wave Equations (Springer, 2001).

15. J.-H. Lee and Q. H. Liu, "An efficient 3-D spectral element method for Schrodinger equation in nanodevice simulation," IEEE Trans. Comput.-Aided Des. 24, 1848-1858 (2005).

16. J.-H. Lee, T. Xiao, and Q. H. Liu, "A 3-D spectral-element method using mixed-order curl conforming vector basis functions for electromagnetic fields," IEEE Trans. Microwave Theory Tech. 54, 437-444 (2006).

17. J.-H. Lee and Q. H. Liu, "A 3-D spectral-element timedomain method for electromagnetic simulation," IEEE Trans. Microwave Theory Tech. 55, 983-991 (2007).

18. A. T. Patera, "A spectral element method for fluid dynamics: Laminar flow in a channel expansion,” J. Comput. Phys. 54, 468-488 (1984).

19. M. Luo, Q. H. Liu, and Z. Li, "Spectral element method for band structures of two-dimensional anisotropic photonic crystals," Phys. Rev. E 79, 026705 (2009).

20. M. Luo and Q. H. Liu, "A spectral element method for band structures of three-dimensional anisotropic photonic crystals," Phys. Rev. E 80, 056702 (2009).

21. M. Luo and Q. H. Liu, "Accurate determination of band structures of two-dimensional dispersive anisotropic photonic crystals by the spectral element method," J. Opt. Soc. Am. A 26, 1598-1605 (2009).

22. P. G. Etchegoin, E. C. Le Ru, and M. Meyer, "An analytic model for the optical properties of gold," J. Chem. Phys. 125, 164705 (2006).

23. P. G. Etchegoin, E. C. Le Ru, and M. Meyer, "Erratum: An analytic model for the optical properties of gold [J. Chem. Phys. 125, 164705 (2006)]," J. Chem. Phys. 127, 189901 (2007).

24. P. S. Light, F. Couny, Y. Y. Wang, N. V. Wheeler, P. J. Roberts, and F. Benabid, "Double photonic bandgap hollow-core photonic crystal fiber,” Opt. Express 17, 16238-16243 (2009).

25. A. D. Rakic, A. B. Djurisic, J. M. Elazar, and M. L. Majewski, "Optical properties of metallic films for vertical-cavity optoelectronic devices,” Appl. Opt. 37, 5271-5283 (1998)

26. S. Y. Lin, J. G. Fleming, D. L. Hetherington, B. K. Smith, R. Biswas, K. M. Ho, M. M. Sigalas, W. Zubrzycki, S. R. Kurtz, and J. Bur, "A three-dimensional photonic crystal operating at infrared wavelengths," Nature 394, 251-253 (1998).

27. S. Noda, K. Tomoda, N. Yamamoto, and A. Chutinan, "Full three-dimensional photonic bandgap crystals at nearinfrared wavelengths," Science 289, 604-606 (2000).

28. B. Gralak, M. de Dood, G. Tayeb, S. Enoch, and D. Maystre, "Theoretical study of photonic band gaps in woodpile crystals," Phys. Rev. E 67, 066601 (2003). 